\title{
Subdiffusion and intermittent dynamic fluctuations in the aging regime of concentrated hard spheres
}

\author{
Djamel El Masri, ${ }^{1,2}$ Ludovic Berthier, ${ }^{2}$ and Luca Cipelletti ${ }^{2}$ \\ ${ }^{1}$ Soft Condensed Matter, Debye Institute for NanoMaterials Science, Utrecht University, \\ Princetonplein 5, 3584 CC Utrecht, The Netherlands \\ ${ }^{2}$ Laboratoire des Colloïdes, Verres et Nanomatériaux, UMR 5587, Université Montpellier 2 and CNRS, 34095 Montpellier, France
}

(Received 23 June 2010; revised manuscript received 26 August 2010; published 24 September 2010)

\begin{abstract}
We study the nonequilibrium aging dynamics in a system of quasihard spheres at large density by means of computer simulations. We find that, after a sudden quench to large density, the relaxation time initially increases exponentially with the age of the system. After a surprisingly large crossover time, the system enters the asymptotic aging regime characterized by a nearly linear increase in the relaxation time with age. In this aging regime, single-particle motion is strongly non-Fickian, with a mean-squared displacement increasing subdiffusively, associated with broad non-Gaussian tails in the distribution of particle displacements. We find that the system ages through temporally intermittent relaxation events, and a detailed finite-size analysis of these collective dynamic fluctuations reveals that these events are not spanning the entire system, but remain spatially localized.
\end{abstract}

DOI: 10.1103/PhysRevE.82.031503

\section{INTRODUCTION}

Aging refers to the slow evolution with time of the physical properties of a disordered material suddenly quenched into a glass phase $[1,2]$. This might refer to the evolution of the density of a polymer glass, of the dielectric response in an organic liquid, or of the height of a gently shaken sandpile. Aging is more easily detected by focusing on the dynamics of the system, for instance, by measuring correlation or response functions. One can think of measuring the decay of density fluctuations using light-scattering techniques in colloidal glasses or the time-dependent magnetic response in a spin glass. After several decades of aging studies in glassy materials, these generic features are very well documented [1-4].

Much less is known, however, about the microscopic mechanisms involved during aging and how these evolve with time for two main reasons. First, the important theoretical developments from the last decade about aging dynamics mainly stemmed from "mean-field" types of approaches, which provided detailed predictions about the behavior of averaged dynamic quantities, but very little information about microscopic motion [2]. Second, it is only relatively recently that the microscopic dynamics of, say, molecules in a supercooled liquid has been characterized in detail in equilibrium conditions above the glass transition [5]; and, by comparison, much less work has been done about the corresponding aging regime at lower temperatures.

In this paper, we use numerical simulations to study the aging dynamics of a model system designed to understand the behavior of dense suspensions of colloidal hard spheres. Hard spheres represent one of the simplest, and thus most studied, models to study the glass transition. It is easily studied in simulations, and the model finds its experimental realization using well-controlled colloidal suspensions [6,7]. In contrast to molecular liquids, colloidal hard spheres can be studied at the particle scale using microscopy techniques $[8,9]$, while dynamic light scattering provides a convenient way to characterize their dynamics in great detail $[10,11]$. It
PACS number(s): 64.70.qd

should be noted that while a relatively small set of experiments have been reported on the aging of colloidal hard spheres [10-14], much broader literature exists on the outof-equilibrium dynamics of a variety of more complex colloidal systems. Throughout this paper, we will in particular make reference to findings for colloidal attractive gels [15-17] and to systems with soft repulsive interactions, such as closely packed soft spheres [18-21] or Laponite clay platelets interacting via Coulomb repulsion [22-27].

In this work, we will be concerned with three main questions:

(1) How does the structural relaxation slow down with the aging time? While numerical simulations of model glasses generically find that the structural relaxation time increases roughly linearly (or sublinearly) with the sample age [28], some light-scattering experiments on gels and Laponite report an unexpected exponential growth of the relaxation time with sample age $[15,22,23]$, at least at short times.

(2) How do particles move during the aging regime? Confocal microscopy experiments report that colloidal particles move very little in concentrated hard spheres to the point that distinguishing thermal vibrations from genuine relaxation becomes an experimental challenge [13,14]. In simulations as well, particles move very little, leading to a very slow, typically algebraic, decay of time correlation functions [28-30]. This is in stark contrast with several experimental reports of sharply decaying time correlation functions in aging molecular liquids seeded with colloidal particles or in colloidal gels and Laponite, where compressed exponential decay and ballistic particle motion over large distances were reported $[4,15,24,31,32]$.

(3) How heterogeneous is the dynamics? Due to the key role played by dynamic heterogeneity in equilibrium studies of the glass transition [5], similar signatures have been sought in aging materials. Optical and confocal microscopy studies revealed the existence of rare small-scale relaxation events involving extended clusters of particles [8], whose size is modest and grows very little [18], or not at all [13,14], during aging. This agrees with numerical simulations on 
model glasses, where four-point dynamic susceptibility appears to grow at a very slow rate with the sample age $[33,34]$, suggesting that if collective displacements occur, they are correlated over relatively short length scales. This set of results is however in contrast with another series of observations. Sudden relaxation events termed (rather dramatically) "earthquakes" [29] or "avalanches" [35,36] were reported in numerical simulations of Lennard-Jones glasses, and these were suggested to be spanning the entire system. Similarly, highly intermittent dynamic fluctuations were experimentally recorded in several aging systems from polymer glasses [37] to colloidal gels [15,16,26] and soft spheres [19], with the latter typically involving ballistic particle motions correlated over very large distances $[16,17,19,20]$.

The paper is organized as follows. In Sec. II, we present the numerical model and techniques used in the present study. In Sec. III, we describe the average aging dynamics for the evolution of the energy and relaxation time scale. In Sec. IV, we focus on the subdiffusive and heterogeneous dynamics at the single-particle level. In Sec. V we present results concerning the intermittent collective dynamics of the system. In Sec. VI we discuss our results and conclude the paper with some perspective for future research.

\section{NUMERICAL MODEL AND TECHNIQUES}

We perform molecular-dynamics simulation of dense systems of strongly repulsive particles interacting with a very steep pair potential designed to model the behavior of colloidal hard spheres [38]:

$$
V\left(r_{i j}\right)=\epsilon\left(\frac{\sigma_{i j}}{r_{i j}}\right)^{36},
$$

where $\epsilon$ is an energy scale; $r_{i j}=\left|\mathbf{r}_{i}-\mathbf{r}_{j}\right|$, with $\mathbf{r}_{i}$ being the position of particle $i$; and $\sigma_{i j} \equiv\left(\sigma_{i}+\sigma_{j}\right) / 2$, where $\sigma_{i}$ represents the diameter of particle $i$. To prevent crystallization occurring in dense systems of hard spheres, we introduce a size polydispersity and draw the particle diameters from a flat size distribution, $\sigma_{i} / \sigma \in[0.8,1.2]$, so that the average diameter is $\overline{\sigma_{i}}=\sigma$, and the polydispersity $\delta$ is given by

$$
\delta=\sqrt{\frac{\overline{\sigma_{i}^{2}}}{\sigma^{2}}-1} \approx 11.5 \% .
$$

To study the dynamics of the system we solve Newton's equations for a system composed of $N$ particles, using a velocity Verlet algorithm [39] in a cubic box of linear size $L$. We use periodic boundary conditions in the three directions of space. We have studied two system sizes: $N=4000$ and $N=500$. When dealing with averages, we shall report results for the largest system size, while a comparison between the two systems will allow us to perform a detailed comparison of the dynamic fluctuations occurring during the aging dynamics as a function of system size.

We perform simulations in the NVT ensemble, and we control the temperature by rescaling velocities every 100 molecular-dynamics time steps in order to maintain the kinetic energy to the desired constant value. Our choice for the frequency of this rescaling results from a compromise: it was chosen to be small enough not to introduce an additional (spurious) slow time scale in the simulations and large enough not to spoil computational efficiency. Note that this technique does not suffer from the shortcomings of the crudest thermostat used in earlier work [28,29], which introduced a strong oscillatory behavior of correlation functions at short times. Finally, we remark that our choice of Newtonian dynamics is not necessarily natural from the point of view of colloidal physics, which would instead suggest the use of Brownian dynamics. Recently, a detailed comparison of the Brownian and Newtonian dynamics of concentrated hard spheres in the aging regime detected no major physical differences between the two approaches [30], extending to the nonequilibrium situations and numerical observations obtained at thermal equilibrium [40,41].

For the inverse power-law potential in Eq. (1), density and temperature cannot be controlled independently, as rescaling the density by a factor $\lambda$ is equivalent to rescaling the temperature by a factor $\lambda^{-1 / 12}$. Thus, we fix the temperature and energy scales, $k_{B} T=\epsilon=1 / 3$, and simply vary the density $\rho$ $=N / L^{3}$ of the system [38]. To ease the comparison with hardsphere experiments, we express our results using the volume fraction, $\varphi$, rather than density $\rho$, where

$$
\varphi=\frac{\pi \rho}{6 N} \sum_{i} \sigma_{i}^{3} .
$$

In the following, we express length scales in units of $\sigma$ and time scales in units of $\tau_{0}=\sigma \sqrt{m / \epsilon}$, where $m$ is the mass of the particles. We use a time discretization $\Delta t=0.01 \tau_{0}$, which ensures a proper integration of the equations of motion.

We shall study the aging dynamics of samples in the range $\varphi=0.553-0.662$. To obtain reproducible results, we need to produce fully disordered initial states. To this end, we first equilibrate the system at a very low volume fraction $\varphi=0.14$. We then compress the system very rapidly to the desired final volume fraction. The compression is done in small successive steps in order to avoid large overlaps leading to very large repulsive forces. The age $t_{\mathrm{w}}$ of the system is counted from the time when the system reaches the final volume fraction. To increase the statistical significance of our results, we have performed five independent runs at each volume fraction with $N=4000$ particles, starting from independent configurations compressed from the fluid at $\varphi$ $=0.14$. During the course of the simulations we found no sign of incipient crystallization in the system, while crystallization was a major obstacle in an initial set of studies using a smaller polydispersity, $\delta \approx 6 \%$, for which we do not present results.

We have previously studied the equilibrium dynamics of this system [11]. We found that the dynamics slows down considerably when $\varphi$ increases above $\varphi \approx 0.50$. Fitting the increase in the relaxation to a power-law divergence at some critical volume fraction $\varphi_{c}$, we obtained $\varphi_{c} \approx 0.592$. This fit then locates the (apparent) mode-coupling singularity for this system [42], which should serve as a reference volume fraction for the aging studies below since it becomes very difficult to reach thermal equilibrium within the accessible numerical time scales when $\varphi$ increases beyond $\varphi_{c}$. 


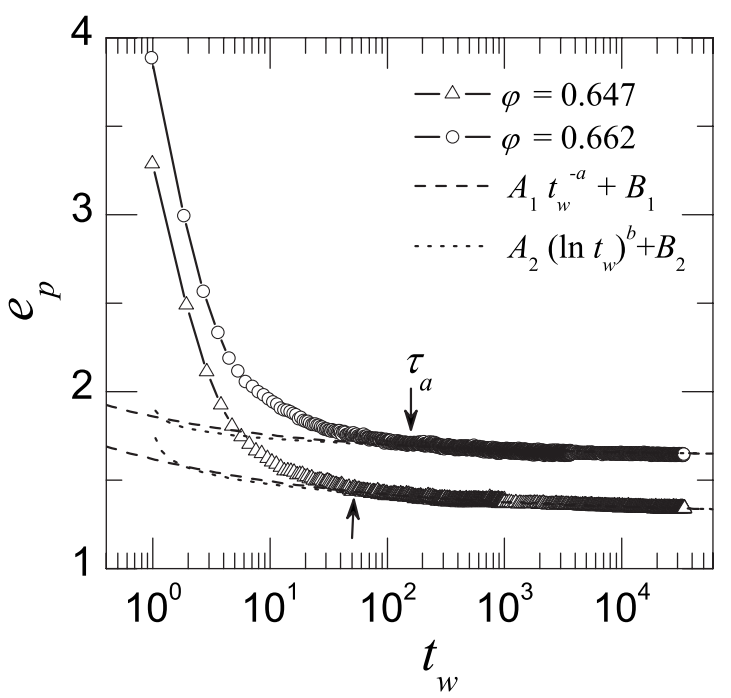

FIG. 1. Aging of the potential-energy density [Eq. (4)] for $\varphi$ $=0.647$ and $\varphi=0.662$. Data are equally well fitted with algebraic, $A_{1} t_{\mathrm{w}}^{-a}+B_{1}$, or logarithmic, $A_{2}\left(\ln t_{\mathrm{w}}\right)^{b}+B_{2}$, time dependence for waiting times larger than a surprisingly large crossover time scale $\tau_{a}$ indicated by arrows.

\section{TOWARD THE ASYMPTOTIC AGING REGIME}

We now present the results of our study, starting from the time evolution of the simplest quantity we can monitor, the potential-energy density, defined as

$$
e_{p}\left(t_{\mathrm{w}}\right)=\left\langle\frac{1}{N} \sum_{i=1}^{N} \sum_{j>i} V\left(r_{i j}\right)\right\rangle,
$$

where the angular brackets stand for an average over independent initial configurations. We present representative results for the time evolution of $e_{p}\left(t_{\mathrm{w}}\right)$ at large densities in Fig. 1.

The central observation from this figure is that, at these large densities, the potential energy keeps evolving over the 6 decades of simulation time and never reaches an asymptotic plateau. This simply confirms that thermal equilibrium cannot be reached at these volume fractions, because the equilibrium relaxation time is much larger than the maximum time accessible to our simulations. More in detail, we also observe that the energy evolves initially quite rapidly, decreasing by a factor of about 2 when time increases from $t_{\mathrm{w}}=1$ to $t_{\mathrm{w}}=10^{2}$, while it evolves only by a few percent when time further increases by two additional decades. Thus, we clearly see the effect of "aging" since dynamical evolution slows down considerably as the age of the system increases, as is well known from decades of experimental aging studies in many different materials.

To describe the slow evolution of the potential energy, we fitted our data to both a power-law decay, $e_{p}\left(t_{\mathrm{w}}\right)=A_{1} t_{\mathrm{w}}^{-a}+B_{1}$, and a logarithmically slow evolution, $e_{p}\left(t_{\mathrm{w}}\right)=A_{2}\left(\ln t_{\mathrm{w}}\right)^{b}+B_{2}$. We find that both fits give an equally good description of the final slow evolution of the potential energy. The fit parameters, $a \approx 0.2-0.3$ and $b \approx 0.1$, confirm that the evolution of the energy is indeed extremely slow.

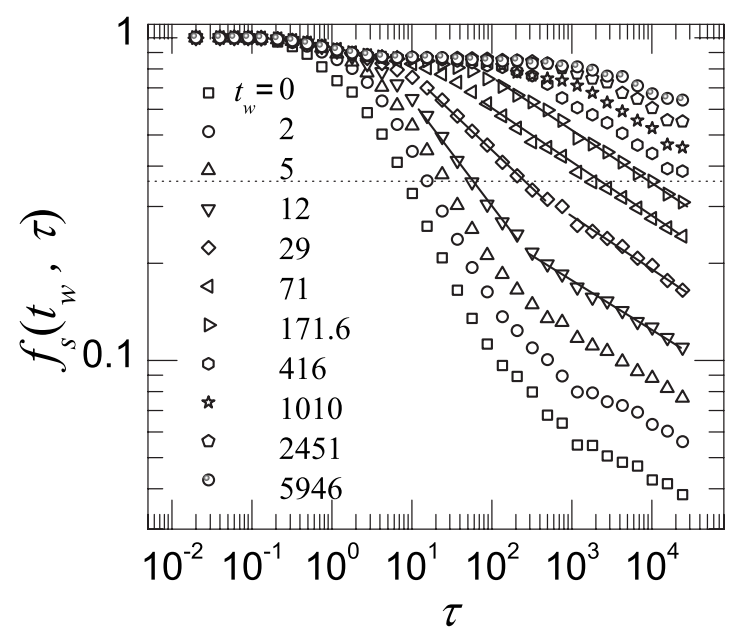

FIG. 2. Self-intermediate scattering function [Eq. (6)] for $\varphi$ $=0.647$ in a log-log representation. The long-time decay is fitted with two power-law decays for $t_{\mathrm{w}}<\tau_{a} \approx 50$, a single one for $t_{\mathrm{w}}$ $>\tau_{a}$, as shown with full lines. The exponent for the latter is nearly constant, $\approx-0.15$. The horizontal dashed line indicates $f_{s}=1 / e$.

We also find that the fits only hold when $t_{\mathrm{w}}$ is very large: the first few decades of the simulation, corresponding to a faster evolution of $e_{p}\left(t_{\mathrm{w}}\right)$, are not well described by this asymptotic slow decay. This implies that a relatively long time is needed for the system to enter the asymptotic aging regime. Moreover, we find that the time $\tau_{a}$ required to enter the asymptotic regime increases when density increases, and the system is quenched deeper into the glassy state. This implies that considerable care must be taken when performing data analysis of the aging regime since it already takes a large part of the simulation simply to enter the final regime, indicated by the arrows in Fig. 1. It is likely that the first regime corresponds to faster processes where the largest overlaps created during the compression are reduced, producing heat which is then removed by the thermostat. Thus, no "universal" characteristics are to be expected in this time regime, which might well depend quite strongly on the details of the preparation procedure or on the chosen thermostat. Note that $\tau_{a} \approx 10^{2} \gg \tau_{0}$ for the data presented in Fig. 1, meaning that scaling behavior might only be observed in the demanding limit of

$$
t_{\mathrm{w}} \gg \tau_{a} \gg \tau_{0} .
$$

It is crucial to recognize the existence of two distinct aging regimes in order to analyze properly the scaling properties of dynamic functions in the asymptotic aging regime, as we find that these two distinct regimes in fact affect most of the measurements we made in our simulations. In Fig. 2 we show the behavior of the self-intermediate scattering function following a quench at $\varphi=0.647$. This two-time quantity is defined as

$$
f_{s}\left(t_{\mathrm{w}}, \tau\right)=\left\langle\frac{1}{N} \sum_{j=1}^{N} e^{i \mathbf{q} \cdot\left[\mathbf{r}_{j}\left(t_{\mathrm{w}}+\tau\right)-\mathbf{r}_{j}\left(t_{\mathrm{w}}\right)\right]}\right\rangle,
$$

and we perform measurements at $q=7.8$, close to the first peak in the static structure factor. As is well known, two-time 


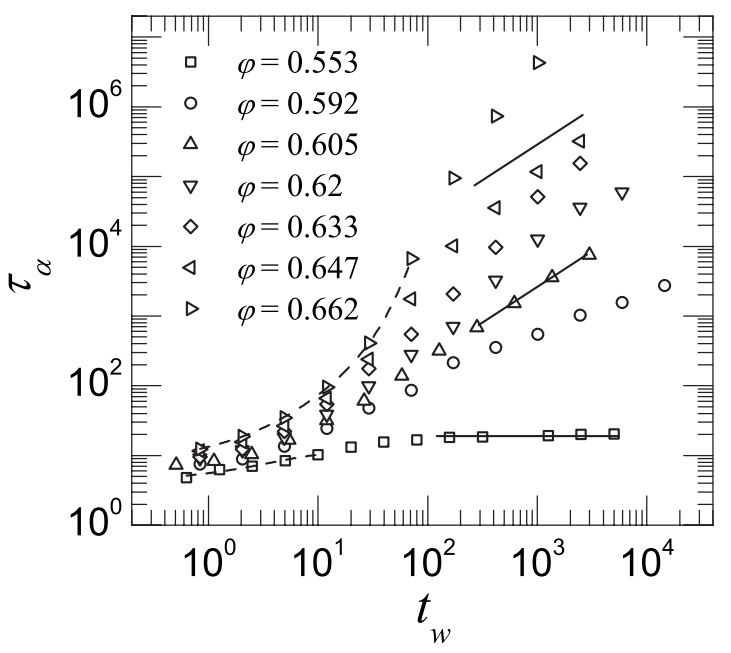

FIG. 3. Evolution of the relaxation time $\tau_{\alpha}\left(t_{w}\right)$ for different volume fractions. The system at $\varphi=0.553$ reaches equilibrium (horizontal line). A simple aging regime $\tau_{\alpha} \sim t_{\mathrm{w}}$, indicated by the full lines, is reached for larger densities, after a transient regime where $\tau_{\alpha}$ grows exponentially with $t_{\mathrm{w}}$, indicated with a dashed line which ends for $t_{\mathrm{w}} \approx \tau_{a}$.

quantities reveal the nonstationary evolution of the system in a very direct manner. In agreement with previous work [28-30], we find that the decay with the delay time $\tau$ of $f_{s}\left(t_{\mathrm{w}}, \tau\right)$ occurs in a two-step manner, with the slow decay being a strong function of the waiting time $t_{\mathrm{w}}$. The faster initial decay is much less sensitive to the waiting time and corresponds physically to particle vibrations in a nearly frozen amorphous structure. Notice that our choice of a thermostat removes the strong short-time oscillations reported in earlier work $[28,29]$.

The existence of two distinct aging regimes is obvious from these data since the slow decay of $f_{s}$ clearly shows a crossover for time delay $\tau$ such that $\tau+t_{\mathrm{w}} \approx \tau_{a} \approx 50$, as shown in Fig. 2, while the data become simpler to describe when $t_{\mathrm{w}}>\tau_{a}$ as the entire decay then takes place in the asymptotic long-time regime defined by Eq. (5). As found in other systems [29], we find that the long-time decay of the self-intermediate scattering function is well described, in the aging regime, by a power law $f_{s}\left(t_{\mathrm{w}}, \tau\right) \sim \tau^{-\alpha}$, as indicated in Fig. 2 by the full lines. As noted before, such an algebraic decay contrasts with equilibrium measurements at lower volume fraction, which typically display stretched exponential relaxations. Stretched exponential decays are only seen for shallow quenches when a crossover toward thermal equilibrium is possible. The small value of the exponent $\alpha \approx 0.15$ means that relaxation occurs over a very broad time window, and that it is not possible to define a "typical" relaxation time for the system.

Nevertheless, to quantify further the aging of the dynamics, we follow the common practice and define an $\alpha$-relaxation time $\tau_{\alpha}\left(t_{\mathrm{w}}\right)$ from the time decay of $f_{s}$ as $f_{s}\left(t_{\mathrm{w}}, \tau_{\alpha}\right)=1 / e$, as indicated by the horizontal dashed line in Fig. 2. In Fig. 3, we report the evolution of $\tau_{\alpha}\left(t_{w}\right)$ with waiting time for all volume fractions investigated in this work, from $\varphi=0.553$ to $\varphi=0.662$. Again, we observe two distinct regimes for the evolution of $\tau_{\alpha}\left(t_{w}\right)$. While for $t_{\mathrm{w}}<\tau_{a}, \tau_{\alpha}$ seems to increase exponentially with waiting time,

$$
\tau_{\alpha} \sim \exp \left(c t_{\mathrm{w}}\right), \quad t_{\mathrm{w}} \ll \tau_{a},
$$

with $c$ being a numerical constant; its growth is better described by an algebraic law at long times, $t_{\mathrm{w}}>\tau_{a}$, which is well compatible with a so-called "simple aging" behavior $[2]$,

$$
\tau_{\alpha} \sim t_{\mathrm{w}}, \quad t_{\mathrm{w}} \gg \tau_{a} .
$$

This simple aging behavior is shown with full lines in Fig. 3, which describes reasonably well, within statistical accuracy, our data from $\varphi=0.605$ up to $\varphi=0.647$. Of course, longer simulations would be required to establish whether Eq. (8) remains true over a wide range of time scales and volume fractions.

In agreement with the observations in Fig. 1, we find that it takes an increasingly long time for the system to enter the asymptotic (simple) aging regime when $\varphi$ increases. For the larger $\varphi$ studied, $\varphi=0.662$, the simple aging regime is not reached during the course of our simulations, and the system appears to be in a crossover between Eqs. (7) and (8), and thus it undergoes an effective "superaging" dynamics, i.e., its relaxation time increases faster than its age. Finally, the opposite "subaging" behavior is found when volume fraction is not very large, for instance, $\varphi=0.592$, because the system is crossing over at large time toward thermal equilibrium, so that $\tau_{\alpha}$ saturates at long waiting times to its equilibrium value. This behavior is in good agreement with the observation of a similar subaging behavior in Ref. [30], where a single volume fraction was studied.

All these observations show that a complex behavior of $\tau_{\alpha}$, as often reported in numerical works $[28,43]$ and experiments on colloidal gels and Laponite $[15,22,23,44]$, may be due to the occurrence of multiple crossovers which are highly sensitive to volume fraction. These observations are also in agreement with the common observation of subaging behavior in aging molecular liquids, for which experiments are traditionally performed not very far below the glass temperature [1,2]. Exponential growth of the relaxation time sometimes (but not always) followed by algebraic growth has been reported in a few experiments on colloidal glasses or gels as well $[15,22,23]$. Our results thus suggest that this exponential growth might well be a transient behavior, which can persist, however, over a very large time window, in particular for very deep quenches. Our results also highlight the difficulty of analyzing the scaling properties of twotime dynamic quantities in numerical simulations, since the asymptotic aging regime is only accessed after a time $\tau_{a}$, which can be very large, thus decreasing the window where universal aging properties can be probed. This might well explain some conflicting results and analyses reported in the literature regarding, e.g., the proper scaling behavior of the self-intermediate scattering function in Lennard-Jones glasses $[45,46]$.

\section{SUBDIFFUSIVE AND HETEROGENEOUS DYNAMICS}

The above findings that the energy decays very slowly while time correlation functions decay in an algebraic man- 


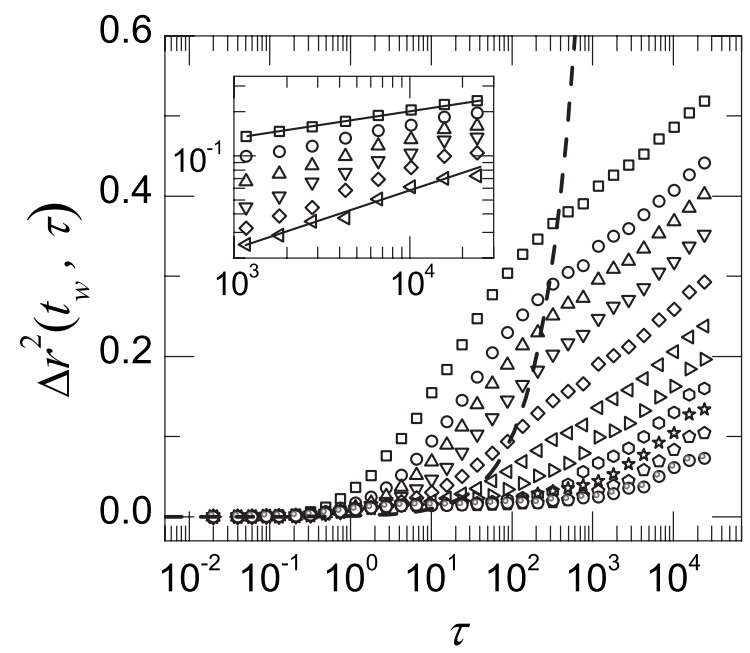

FIG. 4. Mean-squared displacements [Eq. (9)] at $\varphi=0.647$ with symbols as in Fig. 2. The dashed line indicates diffusive behavior. Inset: log-log plot of the data for $t_{\mathrm{w}}>\tau_{a} \approx 50$ and large $\tau$ with subdiffusive fits [Eq. (10)], indicated with full lines.

ner indicate that there is actually very little dynamics taking place in the system. To confirm this notion, we measured the averaged mean-squared displacement,

$$
\Delta r^{2}\left(t_{\mathrm{w}}, \tau\right)=\left\langle\frac{1}{N} \sum_{i=1}^{N}\left|\mathbf{r}_{i}\left(t_{\mathrm{w}}+\tau\right)-\mathbf{r}_{i}\left(t_{\mathrm{w}}\right)\right|^{2}\right\rangle .
$$

In Fig. 4, we present the time evolution of the mean-squared displacements at volume fraction $\varphi=0.647$ measured at different waiting times, using both a standard log-log representation (inset) and a linear-logarithmic representation (main plot). As found in Fig. 2, for the self-intermediate scattering function, the dynamics proceeds again in a two-step fashion with a rapid ballistic regime at short time, followed by vibrations of the particles in a frozen amorphous structure responsible for the plateau behavior at intermediate times observed in Fig. 4. Eventually a much slower waiting-timedependent structural relaxation occurs, which becomes slower when $t_{\mathrm{w}}$ increases. This implies that the aging of the system corresponds, at the microscopic scale, to a dramatic slowing down of single-particle displacements, as found in experiments $[12,13,18]$.

It is significant that in order to represent graphically the behavior of the mean-squared displacements, we had to use a linear scale for its amplitude, with a range which remains smaller than $\Delta r^{2}=0.5$. This implies that over the entire duration of the simulation, particles-on average-move a distance which is smaller than the mean particle diameter: despite the nontrivial aging dynamics we discussed, it should be clear that the structure of the material remains in fact almost frozen as soon as the system is quenched in the glassy phase, with essentially no large-scale dynamics taking place.

The linear representation in Fig. 4 also confirms the existence of the two distinct aging regimes discussed above, thus directly revealing the influence of the time scale $\tau_{a}$ on the dynamics at the particle scale. Again, the data obtained for $t_{\mathrm{w}}<\tau_{a}$ present a crossover for a delay $\tau$ given by

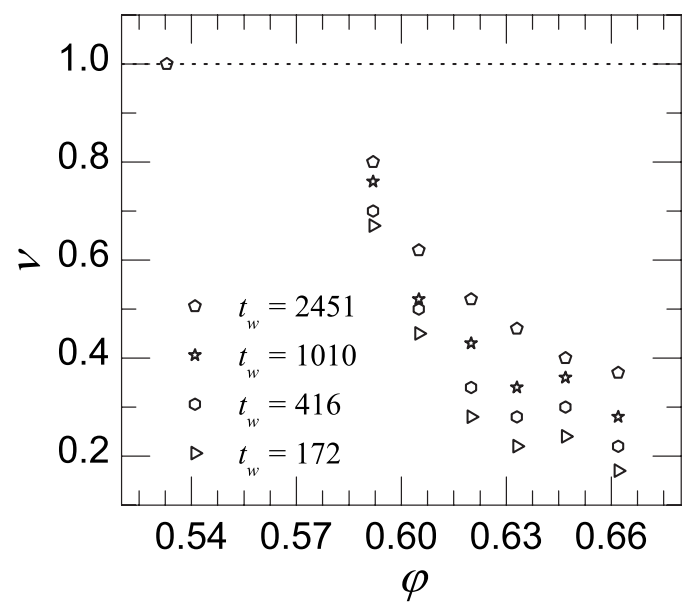

FIG. 5. Evolution of the subdiffusive exponent $\nu$ for various $t_{\mathrm{w}}$ 's and $\varphi$ 's. The exponent deviates further from its equilibrium diffusive limit $\nu=1$ for smaller $t_{\mathrm{w}}$ and larger $\varphi$.

$\tau+t_{\mathrm{w}} \approx \tau_{a} \approx 50$, as seen in Fig. 4. Thus, in the following we again focus on waiting times that are larger than $\tau_{a}$, which we believe to reflect better the universal quench-independent nature of the aging regime in concentrated hard spheres.

In the long-time regime, the dashed line in Fig. 4, which corresponds to a diffusive growth, $\Delta r^{2} \sim \tau$, is obviously an incorrect description of our data since the displacements seem to increase much more slowly than diffusively. As shown in the inset, a subdiffusive law describes our results very satisfactorily,

$$
\Delta r^{2}\left(t_{\mathrm{w}}, \tau\right) \sim \tau^{\nu}, \quad t_{\mathrm{w}} \gg \tau_{a},
$$

where $\nu<1$ is an exponent characterizing the subdiffusion.

We have measured $\nu$ over a broad range of waiting times and volume fractions, and we present its evolution in Fig. 5. Apart from the low volume fractions where (diffusive) thermal equilibrium is reached rapidly, we systematically find that the system obeys subdiffusive rather than diffusive behavior. At a given volume fraction, the system gets closer to equilibrium for larger $t_{\mathrm{w}}$, and correspondingly we find that the exponent $\nu$ increases with the age of the system, although it always remains very far from its equilibrium diffusive value $\nu=1$. Note that an increasing $\nu$ does not imply that particles move faster at large waiting times, as the prefactor in the power law (10) is itself a decreasing function of $t_{\mathrm{w}}$, which implies that the total amplitude of the particle displacements indeed decreases with the age of the system.

As volume fraction increases, the system is quenched deeper and deeper into its glass phase, and deviations from diffusive behavior are correspondingly larger, which translates into a subdiffusive exponent $\nu$ which gets smaller when $\varphi$ increases (see Fig. 5). This simply means that particles move less and less when $\varphi$ becomes larger, which is physically expected.

While the mean-squared displacement quantifies the average dynamical behavior of the system, it does not convey much information on dynamic fluctuations, which are recognized as an important feature of the single-particle dynamics in glassy materials. To quantify this "dynamical heterogene- 


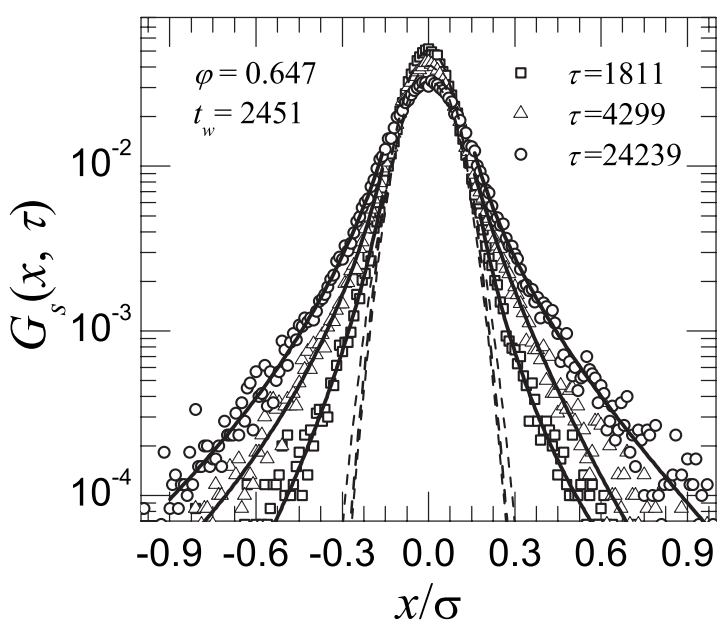

FIG. 6. Distribution of single-particle displacements [Eq. (11)] for $\varphi=0.647, t_{\mathrm{w}}=2451$, and various $\tau$ 's indicated in the figure. While a Gaussian (dashed lines) fits the center of the distribution, the tails are broader and well described by Eq. (12), as shown by the full lines.

ity" during aging, we measure the probability distribution function of the single-particle displacement, also known as the self-part of the Van Hove correlation function:

$$
G_{s}\left(x, t_{\mathrm{w}}, \tau\right)=\left\langle\frac{1}{N} \sum_{i=1}^{N} \delta\left\{x-\left[x_{i}\left(t_{w}+\tau\right)-x_{i}\left(t_{w}\right)\right]\right\}\right\rangle,
$$

where $x_{i}(t)$ represents the projection of $\mathbf{r}_{i}(t)$ along the $x$ axis. To improve the statistics, we use the isotropy of the system and further average $G_{s}\left(x, t_{\mathrm{w}}, \tau\right)$ along the three directions of space. For simplicity, we keep the notation $G_{s}\left(x, t_{\mathrm{w}}, \tau\right)$ for the Van Hove function averaged over the $x, y$, and $z$ directions.

To gain deeper insight into the subdiffusive behavior described above, we present in Fig. 6 the typical shape and evolution of the Van Hove function measured for $\varphi=0.647$, for a large waiting time $t_{\mathrm{w}}=2451$, in the asymptotic aging regime, and a delay $\tau$ within the long-time subdiffusive regime. The data are presented in a linear-logarithmic scale, where Fickian behavior leading to a Gaussian shape of the particle distribution would appear as an inverted parabola, as shown by the dashed lines. These data are highly reminiscent of the equilibrium findings that Van Hove functions are well described at short displacements by a Gaussian form, with tails that are much broader than the Gaussian prediction $[8,9]$. This implies that a small fraction of the particles move significantly farther than what is expected from a purely diffusive and homogeneous process. This is the most basic observation revealing that the system is dynamically heterogeneous and can be described, in a first approximation, as being composed of distinct families of "slow" and "fast" particles. This is a well-established distinctive feature of many disordered glassy materials [5].

How should we describe the functional form of the tails of these distributions? At thermal equilibrium, it is now understood that the tails are well described by an exponential (rather than Gaussian) decay, $G_{s}(x, \tau) \sim \exp (-|x| / \lambda)$, for $\tau$ corresponding to the $\alpha$-relaxation time scale [47]. The physical origin of the exponential, detailed in Refs. [47-49], is due to the stochastic nature of the diffusion process in disordered materials, which is well described by the formalism of continuous time random walks (CTRWs) [50]. In this description, particles in a dense supercooled liquid undergo a succession of long periods of vibrations within the cages formed by their neighbors, followed by rapid "jumps." Both the size of the jumps and, more importantly, the time scale separating them are statistically distributed quantities. The existence of these statistical distributions directly accounts for the exponential form of the tails in the Van Hove functions [47].

The natural extension of these considerations to the nonequilibrium aging regime studied in the present work is the aging continuous time random-walk (ACTRW) formalism [51], whose main features are those of the CTRW recalled above. The only difference lies in the functional form used for the distribution of times separating the jumps, which acquires "fat" non-normalizable tails in the aging regime, in direct analogy with the trap model introduced by Bouchaud to describe aging dynamics in glasses [52]. In this approach, the tails of the jump time distribution decay as $\psi(t) \sim t^{-1-\nu}$, where $\nu<1$ is the subdiffusion exponent introduced in Eq. (10), while the tails of the self-part of the Van Hove function are not exponential anymore, but are instead asymptotically described by [53]

$$
G_{s}\left(x, t_{\mathrm{w}}, \tau\right) \sim|x|^{-\gamma} \exp \left[-(|x| / \lambda)^{\beta}\right],
$$

where the exponents $\gamma$ and $\beta$ are related to the subdiffusion exponent $\nu$; in particular,

$$
\beta=2 /(2-\nu),
$$

so that $\beta=2$ (Gaussian) is recovered when $\nu=1$ (Fickian diffusion). For subdiffusive processes, $0<\nu<1$, one expects instead $1<\beta<2$.

To test in detail the ACTRW picture, one should measure the distribution of jump times $\psi(t)$ and use it to directly predict the mean-squared displacements and Van Hove functions [54]. This is an interesting project, but it lies beyond the scope of the present study. Instead, we more simply use Eq. (12) as a theoretically motivated fitting formula for the tails of the $G_{s}$ distributions. As shown in Fig. 6, such a fit describes the tails very well. We find a similarly good description for a broad range of volume fractions and time scales. Unfortunately, our data are not accurate enough to allow for a very precise determination of all the fitting parameters. Thus, the following results should be discussed at a qualitative, rather than quantitative, level.

In Fig. 7 we present the evolution of the exponent $\beta$ describing the tails of the Van Hove functions in the asymptotic subdiffusive aging regime. We remark that these data have considerably more scatters than the data for the subdiffusive exponent $\nu$, confirming our difficulties to obtain a reliable determination of $\beta$ from the numerical data. Regardless of the noise, we note that $\beta$ values are in the interval $\beta$ $\in[1,2]$, as expected from Eq. (13), suggesting that our description is physically sound. Moreover, the data at large density present a systematic evolution of $\beta$, which increases 


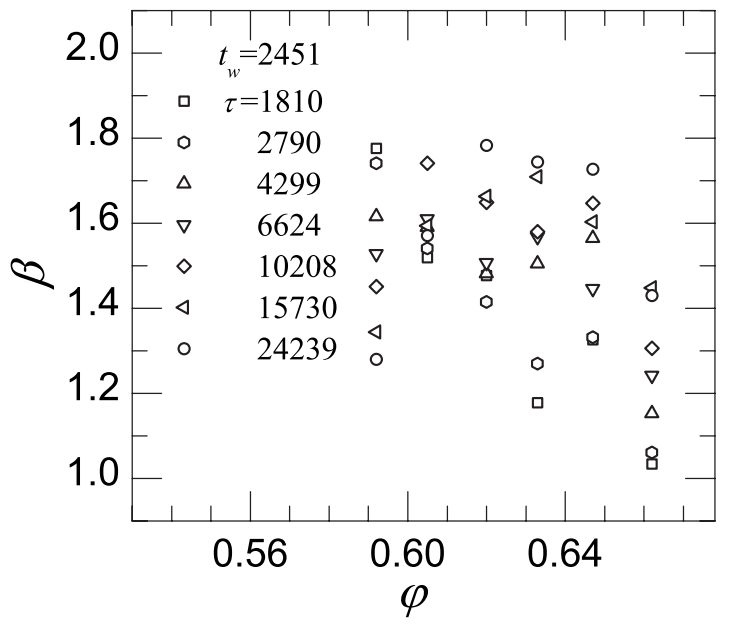

FIG. 7. Evolution of the exponent $\beta$ obtained by fitting the tails of the distribution of particle displacements to Eq. (12), for $t_{\mathrm{w}}$ $=2451$ and several $\tau$ 's and $\varphi$ 's.

toward 2 with $t_{\mathrm{w}}$. Assuming that Eq. (13) is correct, this would imply that $\nu$ increases toward unity with $t_{\mathrm{w}}$, as indeed is observed in Fig. 5. The data at moderate volume fractions, $\varphi<0.60$, are more difficult to interpret as $\beta$ seems to decrease with $t_{\mathrm{w}}$ in this regime, while $\nu$ was found to increase. This could be due to the fact that, for $\varphi=0.592 \approx \varphi_{c}$, the subdiffusive aging regime is crossing over toward equilibrium during the simulation, and so the results could be a subtle combination of the exponential tail reported at intermediate times for equilibrium dynamics [47] and the subdiffusive regime found deeper in the glass and described in the present study.

\section{INTERMITTENT DYNAMIC FLUCTUATIONS}

Our study of single-particle dynamics suggests that particles undergo very little dynamics, leading to a subdiffusive growth of mean-squared displacements, associated with a broad distribution of particle displacements. The picture of ACTRW which we used to describe our data is very similar in spirit to Bouchaud's trap model, and both suggest that the aging dynamics of concentrated hard spheres is a temporally intermittent process [55]. It is the aim of this section to establish whether this picture is indeed correct.

To this end, we must turn to collective dynamic observables. We resolve the aging dynamics in space and time and focus on $\tilde{f}_{s}$, the instantaneous (unaveraged) value of $f_{s}$. We present in Fig. 8(a) the results of independent realizations of the dynamics at a given waiting time $t_{\mathrm{w}}=2451$ and volume fraction $\varphi=0.637$. For this system, containing $N=4000$ particles, we observe small run-to-run fluctuations, which show that resolving the temporal evolution of the dynamics in a system of linear size $L \sim 15 \sigma$ is not sufficient to reveal significant dynamic fluctuations.

Thus, we improve our spatial resolution and repeat this analysis for a smaller system with $L \sim 7.5 \sigma$ which contains $N=500$ particles [see Fig. 8(b)]. The data in Fig. 8 show that run-to-run fluctuations of $\tilde{f}_{s}$ become larger when $N$ is
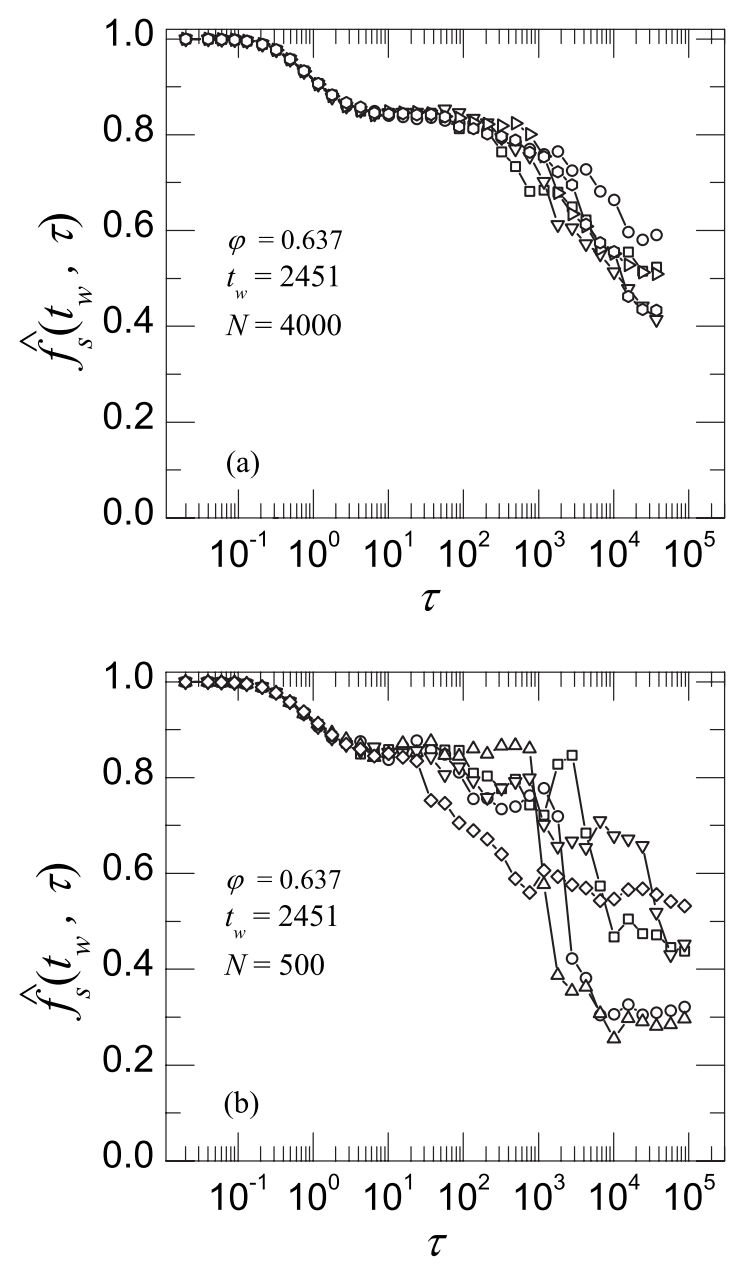

FIG. 8. Instantaneous (unaveraged) intermediate scattering function $\tilde{f}_{s}\left(t_{\mathrm{w}}=2451, \tau\right)$ for the system at $\varphi=0.637$ and five independent quenches, measured in systems with (a) $N=4000$ and (b) $N=500$. While small run-to-run fluctuations are observed in (a), several sudden decorrelation events are observed in (b).

smaller, as expected. However, we emphasize that the nature of the dynamic fluctuations seems to change qualitatively when the size is reduced. For a single realization of a quench with a small enough number of particles, the decay of the self-intermediate scattering function in the long-time regime is highly intermittent, as shown by sudden "drops" of $\tilde{f}_{s}$ [29]. From one run to another, both the temporal location and the amplitude of these drops vary considerably, indicating that in each run the dynamics proceeds via temporally intermittent relaxation events that mobilize a finite fraction of the whole system. However, the comparison with the fluctuations obtained with $N=4000$ suggests that the spatial extension of these intermittent events does not increase with the system size (at least not very rapidly), but instead they tend to remain spatially localized.

Note that this finding could be due to a spurious finite-size effect since an alternative interpretation of the data shown in Fig. 8 could be that sudden decorrelations in the system only occur when the system is too small, and these events are not observed in a bigger system because they disappear altogether. To disprove this interpretation, we reanalyze a single 

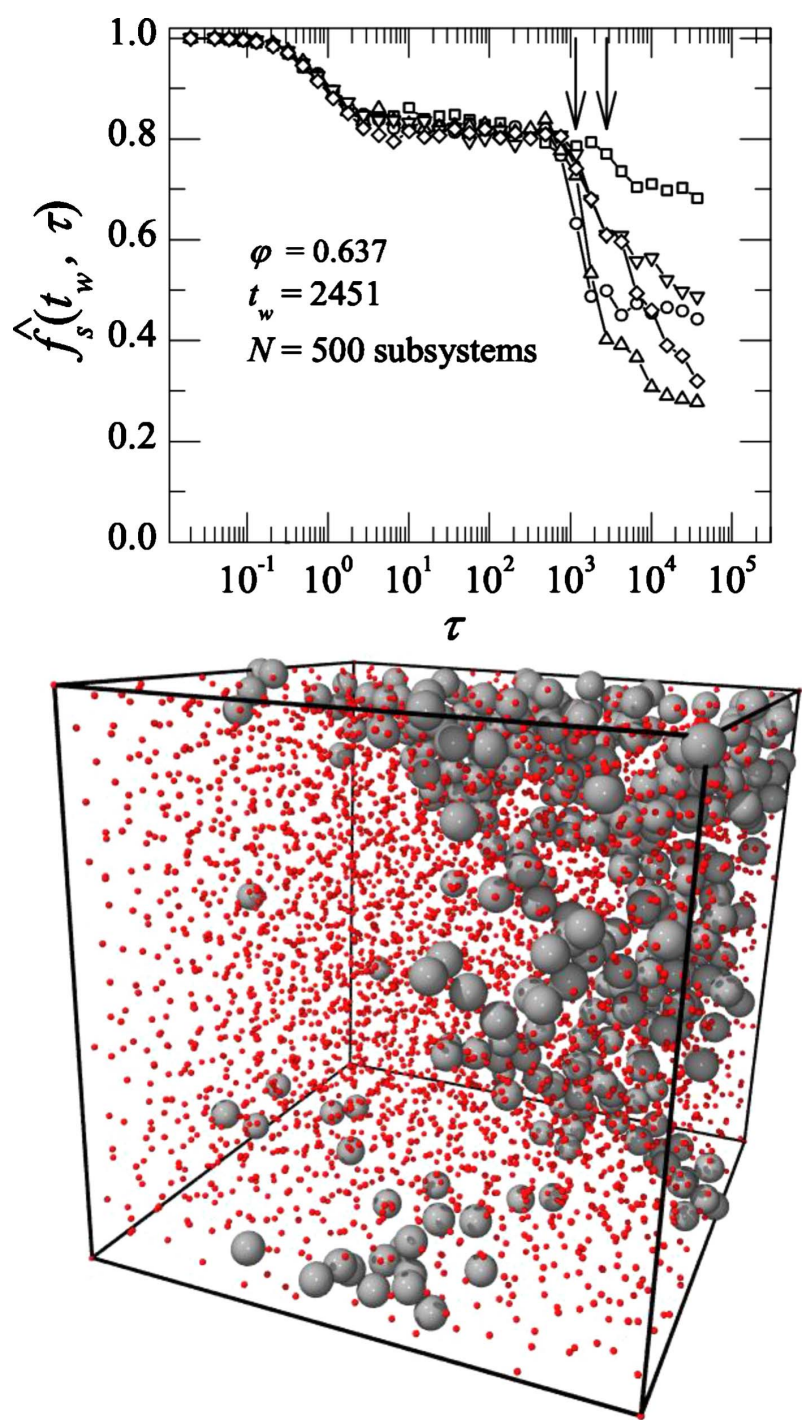

FIG. 9. (Color online) Top: intermediate scattering functions $\tilde{f}_{s}\left(t_{\mathrm{w}}=2451, \tau\right)$ measured in subsystems containing $N=500$ particles within a larger $N=4000$ system. The variability between subsystems in a single realization demonstrates that intermittent decorrelation events are spatially localized. Bottom: snapshot of the $N=4000$ system showing the $5 \%$ most mobile particles over the time interval shown by the two arrows in the top panel $(\tau=1174$ and 2789 , respectively). These most mobile particles, drawn with a larger size, are essentially confined to a corner of the simulation box.

quench with $N=4000$ particles and further decompose the computation of the correlation function in eight distinct subsystems, each comprising 500 particles and corresponding to a cubic sub-box of linear size $L / 2$. In Fig. 9, we present the result of this analysis. Remarkably, we find that in a single quench, some parts of the system might indeed undergo the type of sudden rearrangement seen in $N=500$ particles runs, but in some other parts decorrelation events are not necessarily seen. This is clearly visible in the top graph, where representative $\tilde{f}_{s}$ 's for various sub-boxes are shown: some have large decorrelations while others do not decay. Note moreover the existence of important subsystem-to-subsystem fluctuations, indicating a large degree of independence of the relaxation dynamics within different subsystems of the same sample.

To further illustrate this feature, we highlight in the bottom panel of Fig. 9 those particles that have contributed most to the dynamics between the two times shown by the arrows in top panel. To this end we select particles that have moved by the largest amount, and thus contribute to the nonGaussian tails of the Van Hove distributions shown in Fig. 6. Although the threshold between fast and slow particles is not very sharp, we find that fast particles represent a small fraction of about $4-5 \%$ of all particles, in agreement with previous findings [56]. In the snapshot of Fig. 9, we highlight the $5 \%$ most mobile particles. Clearly, individual rearrangement events are confined to a fraction of the total volume (in that case a corner of the box), thus affecting significantly only the correlation function of the corresponding sub-box. We conclude that in a large system the aging dynamics occurs through an intermittent succession of spatially localized decorrelation events, with no obvious "confinement" effect imposed by the use of a too small system size, at least for the two system sizes used in this study.

Although suggestive, the above finite-size analysis remains qualitative. Therefore, to confirm quantitatively both the absence of serious finite-size effects and the spatially localized nature of decorrelation events, we need to quantify more precisely how extended is the collective aging dynamics in our simulations. To this end, we have measured the variance of the spontaneous fluctuations of the selfintermediate scattering function, also known as a four-point dynamic susceptibility $[33,34]$ :

$$
\chi_{4}\left(t_{\mathrm{w}}, \tau\right)=N\left[\left\langle\hat{f}_{s}^{2}\left(t_{\mathrm{w}}, \tau\right)\right\rangle-\left\langle\hat{f}_{s}\left(t_{\mathrm{w}}, \tau\right)\right\rangle^{2}\right]
$$

where $\hat{f}_{s}$ represents the instantaneous value of $f_{s}$. With this definition, $\chi_{4}$ becomes independent of the system size in the thermodynamic limit, with an amplitude that gives direct access to the typical number of particles relaxing in a correlated manner during aging $[57,58]$. In the presence of finitesize effects, the amplitude of $\chi_{4}$ saturates to a system-sizedependent value [59]. The data for $N=500$ and $N=4000$ shown in Fig. 10 are essentially the same, within our statistical accuracy, for both system sizes, and $\chi_{4}$ reaches a value near $\chi_{4} \approx 10$ for time scales corresponding to the slow relaxation of the correlation function, thus confirming the absence of finite-size effects in our data, which physically implies that the relevant relaxation events are spatially localized in our system. It is interesting to note that $\chi_{4}$ peaks at a modest value of about 10-20. A possible interpretation is that intermittent relaxation events involving a larger (but finite) number of particles, as depicted in the snapshot in Fig. 9, are also rather sparse and sporadic.

\section{DISCUSSION}

We studied numerically the aging dynamics of a concentrated system of nearly hard spheres over a broad range of volume fractions and a large time window. We addressed the three central questions presented in the introduction, concerning the evolution of the dynamics, single-particle motion, and the heterogeneous nature of the dynamics. 


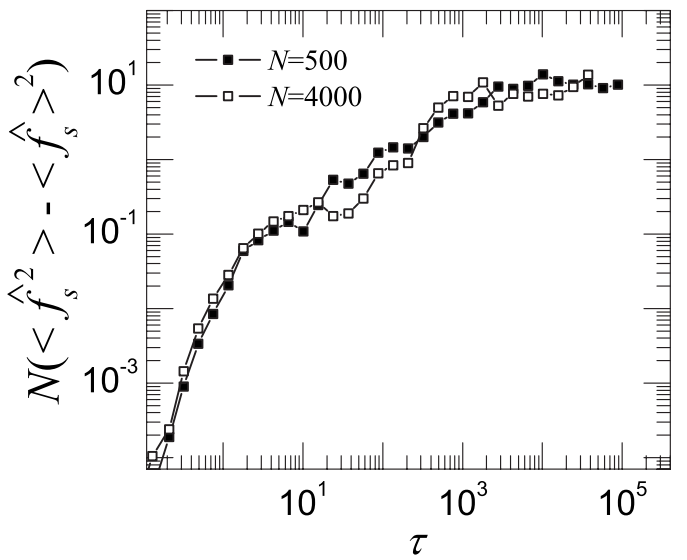

FIG. 10. The four-point susceptibility $\chi_{4}$ [Eq. (14)] for $t_{\mathrm{w}}$ $=2541, \varphi=0.637$, and two system sizes shows no system size dependence, and its modest peak value, $\chi_{4} \approx 10$, confirms the localized nature of decorrelation events.

We found that over the 6 decades of aging time covered by our simulations, the structural relaxation continuously slows down, as expected for any aging system, but we showed that an asymptotic aging regime is only accessed after a time scale $\tau_{a}$, which can become quite large for dense systems. Interestingly, we found that the relaxation time $\tau_{\alpha}\left(t_{\mathrm{w}}\right)$ first increases exponentially, as observed in some experiments on colloidal gels and repulsive Laponite platelets $[15,22,23]$, before crossing over to a form compatible with simple aging, $\tau_{\alpha} \sim t_{\mathrm{w}}$, at large times $t_{\mathrm{w}} \gg \tau_{a}$. In the initial regime, we also found that the energy density decays very quickly, suggesting that the system evolves rapidly from its highly disordered initial state to form a nearly frozen structure, which then ages more slowly. Thus, this initial regime is likely quite sensitive to the detailed initialization procedure of the system both in simulations and experiments. The universal features of the aging dynamics of concentrated hard spheres should be discussed for the second regime only, and our simulations suggest that, once care has been taken of the above-mentioned crossovers, concentrated hard spheres follow a simple aging form, as indeed found for very many glassy materials $[1,2]$. At lower volume fractions, subaging behavior can be observed because the dynamics eventually crosses over toward equilibrium.

In the aging regime, we found that particle motions are very restrained, with particles moving on average less than their own diameters over the entire duration of the simulations. In the aging regime, $t_{\mathrm{w}} \gg \tau_{a}$, we found that the selfintermediate functions decay algebraically at long times, in agreement with several simulations of soft and hard particle systems [28-30]. This is also in agreement with recent experiments performed on colloidal suspensions that are dense enough, so that the aging regime does not cross over at long times toward equilibrium behavior $[10,11]$.

We also found that single-particle motion is subdiffusive, $\Delta r^{2}\left(t_{\mathrm{w}}, \tau\right) \sim \tau^{\nu}$, with $\nu<1$. This behavior is actually quite consistent with the results obtained by optical and confocal microscopy on hard and soft colloids, where diffusive behavior is not reached in the experimental time window $[13,14,18]$. This result is also in broad agreement with earlier numerical work [28], although subdiffusion was never described in detail before. We have suggested that a natural theoretical framework to analyze our results should be the ACTRW, i.e., the nonequilibrium extension of the randomwalk picture used to describe single-particle motion at thermal equilibrium near the glass transition $[47,48]$. This formalism makes a number of detailed predictions for the aging regime $[51,55]$. Here, because of a lack of statistics, we were only able to show that our data are in qualitative agreement with this picture, which can link the shape of the distribution of particle displacements to the subdiffusion exponent. It would be extremely interesting to extend this analysis in future work and check in more detail how far the ACTRW picture can be pushed to describe the aging dynamics of concentrated hard spheres.

Finally, by resolving the measurement of time correlation functions in space and time, we showed that the aging dynamics of concentrated hard spheres is highly intermittent, with very sudden relaxation events separating at long periods of time where very little dynamics occurs. Similar dynamic events have been observed in numerical work before and were coined earthquakes [29] or avalanches [36]. Although these names and additional numerical evidence suggested that these events could well be system spanning [36], we showed by using much larger system sizes that these events remained actually spatially localized and do not grow with system size. This is in fact quite consistent with the experimental report of dynamic clusters that grow rather modestly in aging colloidal samples $[14,18]$, and with numerical work reporting a slow growth of four-point dynamic susceptibilities in aging Lennard-Jones glasses [34].

Thus, we find that in concentrated hard spheres the dynamics are intermittent as it occurs in several more complex colloidal materials, but this leads neither to anomalous compressed exponential relaxation for time correlation functions [15] nor to ballistic motion over large distances $[15,17,19,20,60]$, whose origin thus remains largely mysterious. This means that model systems such as hard spheres or Lennard-Jones glasses are not good starting points to gain insight into the nature of these intriguing aging dynamics. It is not clear what ingredient these models are missing since similar anomalous aging dynamics was recently reported in simple hard molecular glasses approaching the glass transition $[31,32]$. It would be very interesting to discover a model system displaying the same type of anomalous aging dynamics, with a particle-scale dynamics that can be followed in the simulations in the way similar to what we did in the present paper for hard spheres.

\section{ACKNOWLEDGMENTS}

This work was financed by Grants NWO-SRON, ANR Dynhet, Région Languedoc-Roussillon "Chercheurs d'avenir," and ACI Jeunes Chercheurs. 
[1] L. C. E. Struik, Physical Aging in Amorphous Polymers and Other Materials (Elsevier, Amsterdam, 1978).

[2] Spin Glasses and Random Fields, edited by A. P. Young (World Scientific, Singapore, 1998).

[3] Slow Relaxations and Nonequilibrium Dynamics in Condensed Matter, edited by J.-L. Barrat, J. Dalibard, M. Feigelman, and J. Kurchan (Springer, Berlin, 2003).

[4] L. Cipelletti and L. Ramos, J. Phys.: Condens. Matter 17, R253 (2005)

[5] M. D. Ediger, Annu. Rev. Phys. Chem. 51, 99 (2000).

[6] P. N. Pusey and W. van Megen, Nature (London) 320, 340 (1986).

[7] E. Weeks and L. Cipelletti (unpublished).

[8] E. Weeks, J. C. Crocker, A. C. Levitt, A. Schofield, and D. A. Weitz, Science 287, 627 (2000).

[9] W. K. Kegel and A. van Blaaderen, Science 287, 290 (2000).

[10] V. A. Martinez, G. Bryant, and W. van Megen, Phys. Rev. Lett. 101, 135702 (2008).

[11] D. El Masri, G. Brambilla, M. Pierno, G. Petekidis, A. Schofield, L. Berthier, and L. Cipelletti, J. Stat. Mech.: Theory Exp. (2009), P07015.

[12] N. B. Simeonova and W. K. Kegel, Phys. Rev. Lett. 93, 035701 (2004).

[13] R. E. Courtland and E. R. Weeks, J. Phys.: Condens. Matter 15, S359 (2003).

[14] J. M. Lynch, G. C. Cianci, and E. R. Weeks, Phys. Rev. E 78, 031410 (2008).

[15] L. Cipelletti, S. Manley, R. C. Ball, and D. A. Weitz, Phys. Rev. Lett. 84, 2275 (2000).

[16] A. Duri and L. Cipelletti, EPL 76, 972 (2006).

[17] A. Duri, D. A. Sessoms, V. Trappe, and L. Cipelletti, Phys. Rev. Lett. 102, 085702 (2009).

[18] P. Yunker, Z. Zhang, K. B. Aptowicz, and A. G. Yodh, Phys. Rev. Lett. 103, 115701 (2009).

[19] S. Mazoyer, L. Cipelletti, and L. Ramos, Phys. Rev. Lett. 97, 238301 (2006).

[20] S. Mazoyer, L. Cipelletti, and L. Ramos, Phys. Rev. E 79, 011501 (2009).

[21] D. van den Ende, E. H. Purnomo, M. H. G. Duits, W. Richtering, and F. Mugele, Phys. Rev. E 81, 011404 (2010).

[22] B. Abou, D. Bonn, and J. Meunier, Phys. Rev. E 64, 021510 (2001).

[23] S. Kaloun, R. Skouri, M. Skouri, J. P. Munch, and F. Schosseler, Phys. Rev. E 72, 011403 (2005).

[24] R. Bandyopadhyay, D. Liang, H. Yardimci, D. A. Sessoms, M. A. Borthwick, S. G. J. Mochrie, J. L. Harden, and R. L. Leheny, Phys. Rev. Lett. 93, 228302 (2004).

[25] Y. M. Joshi and G. R. K. Reddy, Phys. Rev. E 77, 021501 (2008).

[26] A. Mamane, C. Fretigny, F. Lequeux, and L. Talini, EPL 88, 58002 (2009).

[27] N. Greinert, T. Wood, and P. Bartlett, Phys. Rev. Lett. 97, 265702 (2006).

[28] W. Kob and J.-L. Barrat, Phys. Rev. Lett. 78, 4581 (1997).

[29] W. Kob and J.-L. Barrat, Eur. Phys. J. B 13, 319 (2000).
[30] A. M. Puertas, J. Phys.: Condens. Matter 22, 104121 (2010).

[31] C. Caronna, Y. Chushkin, A. Madsen, and A. Cupane, Phys. Rev. Lett. 100, 055702 (2008).

[32] H. Guo, G. Bourret, M. K. Corbierre, S. Rucareanu, R. B. Lennox, K. Laaziri, L. Piche, M. Sutton, J. L. Harden, and R. L. Leheny, Phys. Rev. Lett. 102, 075702 (2009).

[33] G. Parisi, J. Phys. Chem. B 103, 4128 (1999).

[34] A. Parsaeian and H. E. Castillo, Phys. Rev. E 78, 060105(R) (2008).

[35] K. Vollmayer-Lee, W. Kob, K. Binder, and A. Zippelius, J. Chem. Phys. 116, 5158 (2002).

[36] K. Vollmayer-Lee and E. A. Baker, EPL 76, 1130 (2006).

[37] L. Buisson, L. Bellon, and S. Ciliberto, J. Phys.: Condens. Matter 15, S1163 (2003).

[38] T. Voigtmann, A. M. Puertas, and M. Fuchs, Phys. Rev. E 70, 061506 (2004).

[39] M. Allen and D. Tildesley, Computer Simulation of Liquids (Oxford University Press, Oxford, 1987).

[40] T. Gleim, W. Kob, and K. Binder, Phys. Rev. Lett. 81, 4404 (1998)

[41] L. Berthier and W. Kob, J. Phys.: Condens. Matter 19, 205130 (2007).

[42] G. Brambilla, D. El Masri, M. Pierno, L. Berthier, L. Cipelletti, G. Petekidis, and A. B. Schofield, Phys. Rev. Lett. 102, 085703 (2009).

[43] K. Vollmayr-Lee, J. A. Roman, and J. Horbach, Phys. Rev. E 81, 061203 (2010).

[44] A. S. Negi and C. O. Osuji, Phys. Rev. E 80, 010404 (2009).

[45] U. Müssel and H. Rieger, Phys. Rev. Lett. 81, 930 (1998).

[46] W. Kob and J.-L. Barrat, Phys. Rev. Lett. 81, 931 (1998).

[47] P. Chaudhuri, L. Berthier, and W. Kob, Phys. Rev. Lett. 99, 060604 (2007).

[48] L. Berthier, D. Chandler, and J. P. Garrahan, EPL 69, 320 (2005).

[49] P. Chaudhuri, Y. Gao, L. Berthier, M. Kilfoil, and W. Kob, J. Phys.: Condens. Matter 20, 244126 (2008).

[50] E. W. Montroll and G. H. Weiss, J. Math. Phys. 6, 167 (1965).

[51] E. Barkai and Y. Cheng, J. Chem. Phys. 118, 6167 (2003).

[52] J. P. Bouchaud, J. Phys. I 2, 1705 (1992).

[53] R. Metzler and J. Klafter, Phys. Rep. 339, 1 (2000).

[54] M. Warren and J. Rottler, EPL 88, 58005 (2009).

[55] C. Monthus and J.-P. Bouchaud, J. Phys. A 29, 3847 (1996).

[56] W. Kob, C. Donati, S. J. Plimpton, P. H. Poole, and S. C. Glotzer, Phys. Rev. Lett. 79, 2827 (1997).

[57] C. Toninelli, M. Wyart, L. Berthier, G. Biroli, and J.-P. Bouchaud, Phys. Rev. E 71, 041505 (2005).

[58] C. Dalle-Ferrier, C. Thibierge, C. Alba-Simionesco, L. Berthier, G. Biroli, J.-P. Bouchaud, F. Ladieu, D. L'Hôte, and G. Tarjus, Phys. Rev. E 76, 041510 (2007).

[59] L. Berthier, Phys. Rev. Lett. 91, 055701 (2003).

[60] S. Maccarrone, G. Brambilla, O. Pravaz, A. Duri, M. Ciccotti, J.-M. Fromental, E. Pashkovski, A. Lips, D. Sessoms, V. Trappe, and L. Cipelletti, e-print arXiv:1006.5527, Soft Matter (to be published). 